\title{
A Flexible Millimeter Wave Radio Channel Emulator Design with Experimental Validations
}

\author{
Wei Fan, Pekka Kyösti, Lassi Hentilä, and Gert F. Pedersen
}

\begin{abstract}
Millimeter wave (mmWave) channel emulator (CE) is an essential tool for air interface testing of the upcoming mmWave communication systems. A flexible $\mathrm{CE}$, which is capable of frequency setting from sub-6 $\mathrm{GHz}$ to mmWave bands and flexible system bandwidth setting, is highly desirable. This is due to the fact that potential frequency bands and system bandwidths are undecided for mmWave systems and it is likely that multibands with scalable system bandwidths will be supported. In this paper, a frequency extension scheme based on frequency up-anddown conversion, and a novel bandwidth enhancement scheme based on the band-combining principle, are proposed to upgrade existing sub-6GHz CE to meet the requirements in the mmWave $\mathrm{CE}$ design. The designed mmWave $\mathrm{CE}$ is experimentally validated, where the measured Doppler and delay profiles agree well with the target ones. Moreover, the beamforming validation measurements showed that excellent gain and phase control of the mmWave $\mathrm{CE}$ output ports can be realized.
\end{abstract}

Index Terms-Multipath channels, radio propagation, testing

\section{INTRODUCTION}

Utilization of millimeter wave (mmWave) frequencies for the fifth generation $(5 \mathrm{G})$ has been a hot research topic in recent years. The reasons can be attributed to the huge available frequency spectrum in mmWave bands [1]. Strong efforts have been taken to investigate potential new bands for $5 \mathrm{G}$, where various frequency bands from the $6 \mathrm{GHz}$ to $100 \mathrm{GHz}$ have been proposed [1]. It is difficult to assess frequency bands and required bandwidths for $5 \mathrm{G}$ mmWave cellular deployment at current stage. It is typically expected that orders of magnitude larger bandwidth should be made available for 5G, compared to current sub-6GHz cellular systems, to meet the high datarate requirement [1]. Similar to sub- $6 \mathrm{GHz}$ cellular systems, it is likely that multiple frequency bands with various system bandwidths will be adopted in $5 \mathrm{G}$ cellular systems [1].

The radio channel emulator (CE), which is typically used to emulate the radio channel between the transmitter (Tx) and the receiver $(\mathrm{Rx})$, is an essential component for air interface testing in wireless communication [2]. In the laboratory environment, real-world radio environment can be mathematically modeled and physically implemented in the CE. Therefore, radio $\mathrm{CE}$ is seen as an essential tool that can facilitate the test and validation cycles of wireless communication systems [2].

The radio $\mathrm{CE}$ has been used to emulate the propagation channels and other system components (if can be mathematically modeled) for various purposes. For example, in

Wei Fan, and Gert F. Pedersen are with the Antennas, Propagation and Millimeter-Wave Systems (APMS) section at the Department of Electronic Systems, Aalborg University, Denmark (email: \{wfa\}@es.aau.dk).

Pekka Kyösti and Lassi Hentilä are with Keysight Technologies, Finland.

Pekka Kyösti is also with center for wireless communications (CWC), Oulu university, Oulu, FI-90014, Finland. traditional cable testing, the BS and mobile terminal antenna characteristics, if known, can be included in the radio channel models implemented in the CE [3]. For over-the-air (OTA) testing in the multi-probe anechoic chamber (MPAC) setups, the focus has been on reconstructing the spatial profiles at the DUT side by allocating weighted channel profiles to the OTA antennas [4]. For the radiated two-stage method (aka. wireless cable method), transfer matrix between the OTA antenna and DUT antenna can be measured and then calibrated out in the radio CE [3], [5], [6]. It was described in [7], [8] that antenna arrays at the BS and mobile terminal side, along with the propagation channels, can be emulated in the CE. In [9], it was proposed to mathematically model the MPAC environment in the $\mathrm{CE}$ for concept validation. However, current commercial radio CEs are generally designed for sub- $6 \mathrm{GHz}$ applications, with a limited system bandwidth. For example, commercial sub 6-GHz CEs with a maximal supported bandwidth of 50 $\mathrm{MHz}, 100 \mathrm{MHz}$ and $160 \mathrm{MHz}$ are reported in [10], [11] and [12], respectively.There is a strong need to design a CE that is suitable for mmWave applications. mmWave CE design has recently attracted huge attention both from the academia and industry, due to its important role in wireless device testing. A commercial frequency converter is presented in [13] to enable channel emulation from traditional sub- $6 \mathrm{GHz}$ range to mmWave range of $27.5 \mathrm{GHz}$ to $28.5 \mathrm{GHz}$ and vice versa. A mmWave CE design based on software define ratio (SDR) platform with $3 \mathrm{GHz}$ of instantaneous bandwidth was reported in [8], though no details were given.

In this paper, a flexible mmWave $\mathrm{CE}$ design based on an existing sub- $6 \mathrm{GHz} \mathrm{CE}$ is proposed and experimentally validated. The system cost can be significantly reduced if a mmWave CE can be realized based on available CE. To the best knowledge of the authors, this has not yet been reported in the literature. The main contributions of the paper lie in:

- To cover channel emulation both for sub-6GHz and mmWave applications, a flexible carrier frequency extension scheme based on frequency converters is utilized.

- To achieve flexible CE instantaneous bandwidth, a novel band-combining scheme is proposed. Further, a lowpass filter design for each sub-band channel and system calibration for the band-combining scheme are discussed.

- To investigate to what degree we can accurately emulate channel models based on the proposed mmWave $\mathrm{CE}$ design, we experimentally validated both the channel Doppler and delay profiles in a practical setup.

- Phase and gain control at CE output port are important for many applications, e.g. system calibration, channel 


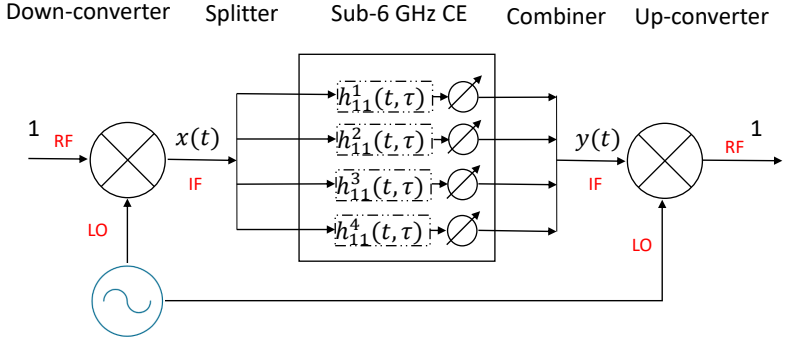

Figure 1. A diagram of the carrier frequency extension scheme and bandwidth extension scheme. Note that the carrier frequency extension and bandwidth enhancement principles are detailed in Section II. A and II. B, respectively.

emulation in OTA testing [4], [6]. To validate the complex weight accuracy of the designed mmWave CE, a beamforming validation measurement is presented.

\section{System Design}

\section{A. Extension to mmWave frequency band}

To extend the frequency range to mmWave bands based on existing sub-6 $\mathrm{GHz}$ CEs, we can utilize frequency mixers to up-convert and down-convert signal frequency, as illustrated in Fig. 1. The RF frequency $f_{\mathrm{RF}}$ is calculated as:

$$
f_{\mathrm{RF}}=f_{\mathrm{LO}}-f_{\mathrm{IF}},
$$

where $f_{\mathrm{LO}}$ is the local oscillator (LO) frequency and $f_{\mathrm{IF}}$ is the carrier frequency setting in the sub- $6 \mathrm{GHz} \mathrm{CE}$, respectively. $f_{\mathrm{IF}}=5.1 \mathrm{GHz}$ and $f_{\mathrm{LO}}=33.1 \mathrm{GHz}$ are set to achieve $f_{\mathrm{RF}}=$ $28 \mathrm{GHz}$ as an example. mmWave carrier frequency $f_{\mathrm{RF}}$ can be flexibly set via setting $f_{\mathrm{LO}}$ and $f_{\mathrm{IF}}$.

\section{B. Bandwidth enhancement scheme}

1) Band-combining scheme: A novel $\mathrm{CE}$ bandwidth enhancement scheme based on band-combining principle is proposed as illustrated in Fig. 1, where $h_{i j}^{n}(t, \tau)$ denotes the channel impulse response (CIR) between the $i$-th $(i \in[1, I])$ mmWave CE output port and the $j$-th $(j \in[1, J])$ input port, and the superscript index $n$, if any, indicates the $n$-th sub-band. A band-combining scheme can be realized, with the help of a power splitter, multiple fading units (with supported bandwidth $B_{s}$ ) within the $\mathrm{CE}$, and a power combiner, as illustrated in Fig. 1. In the following, the band-combining scheme is described for a single fading channel (i.e. $I=J=1$ ) for notational simplicity (as shown in Fig. 1), though its principle is applicable to MIMO fading channels. The band-combining scheme is illustrated in Fig. 2 and detailed below:

1) Down-convert the mmWave Tx signal to obtain $x(t)$.

2) Split $x(t)$ to $N$ identical branches $x^{n}(t), n \in[1, N]$.

3) Route each branch to $n=1, \ldots, N$ fading unit. Each fading unit has been set a unique center frequency $f^{n}$

$$
f^{n}=f_{I F}+\left(n-\frac{N+1}{2}\right) B_{o},
$$

where $B_{O}$ is the target bandwidth to be achieved.
4) Down-convert frequency from $f^{n}$ to base-band for $x(t)$ of the $n$-th fading unit to obtain the base-band signal $x_{b}^{n}(t), n \in[1, N]$, where subscript $b$ denotes baseband.

5) Perform low-pass filtering for $x_{b}^{n}(t)$

$$
x_{l p}^{n}(t)=x_{b}^{n}(t) * g(\tau),
$$

where $g(\tau)$ is the low-pass filter to be discussed, and subscript $l p$ denotes low-pass.

6) Perform frequency shifting of the original CIR over target band $B_{o}$ in the $n$-th fading unit

$$
h_{b}^{n}(t, \tau)=h(t, \tau) \exp \left(i 2 \pi \tau\left(f_{I F}-f^{n}\right)\right)
$$

7) Perform convolution operations to obtain base-band (faded) output signals

$$
y_{b}^{n}(t)=x_{l p}^{n}(t) * h_{b}^{n}(t, \tau) .
$$

8) Up-convert frequency from base-band to $f^{n}$ for $y_{b}^{n}(t)$.

9) Route signals to CE output ports and combine the signal $y(t)=\Sigma_{n} y^{n}(t)$.

10) Up-convert $y(t)$ to RF in the mmWave band.

Basically, we take advantage of the parallelization of signal processing to extend the system bandwidth. Separate fading units can be used to perform fading of respective frequency band slices of the Tx signal. It is noted that we have to trade off CE resource (e.g. interface ports and fading units) for more system bandwidth in the band-combining scheme.

2) Low-pass filter design: The low-pass filter $g(\tau)$ has to be designed to support for seamless combination of subbands. The filter has to fulfill the Nyquist pulse shaping criterion [14]. A sinc filter is an ideal low-pass filter, where the bandwidth for each subband is $B_{s}=B_{o} / N$ without overlapping between sub-bands. An ideal filter which offers a "brick wall" frequency response, however, does not exist in practical applications since it is non-casual and has an infinite delay. We can utilize the overlapping low-pass filters (i.e. $B_{s}>B_{o} / N$ ) instead [14]. A low-pass filter of particular interest and has been widely used in practice to achieve desirable spectral properties is the raised cosine spectrum [14]:

$$
g(\tau)=\operatorname{sinc}(\tau / T) \frac{\cos (\pi \beta \tau / T)}{1-4 \beta^{2} \tau^{2} / T^{2}},
$$

where $T$ is the sampling interval and $\beta \in[0,1]$ is the rolloff factor. Typically, when designing the band combining low-pass filter, one has to balance between the available computing resources (i.e. number of filter taps), the target useful bandwidth, and the stop band attenuation requirements. An example of the filter design is illustrated in Fig. 3.

3) Calibration procedure: As can been seen in Fig. 1, the RF chain associated with each sub-band in the CE is actually different in the practical system. Therefore, we might expect unequal frequency responses of the RF chains, which would deteriorate the performance of the band-combining scheme. This can be overcome by tuning the gain and phase at the sub6GHz CE output port for each sub-band RF chain, as shown in Fig. 1. The calibration procedure and results are further explained in the Section III-B for a practical system. 

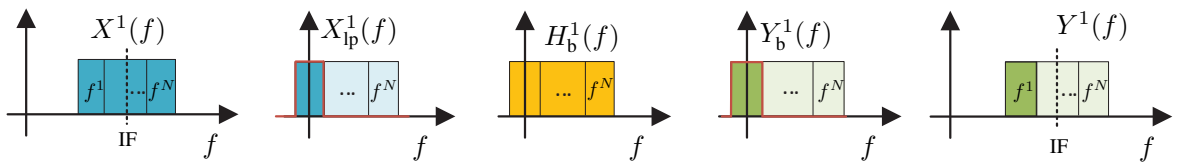

$\vdots n$

:n
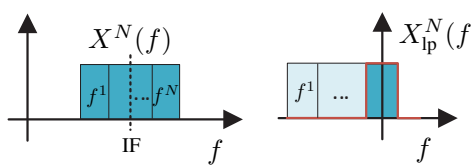

:n
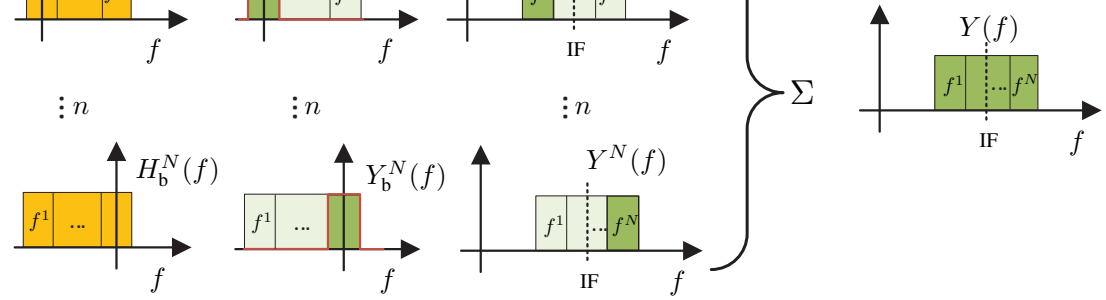

(2)-(3)

(4)-(5)

(6)

(7)

(8)

(9)

Figure 2. An illustration of the band-combining procedure in the sub-6GHz CE. $X^{n}(f)$ and $x^{n}(t), X_{l p}^{n}(f)$ and $x_{l p}^{n}(t), H_{b}^{n}(f)$ and $h_{b}^{n}(t), Y_{b}^{n}(f)$ and $y_{b}^{n}(t), Y^{n}(f)$ and $y^{n}(t), Y(f)$ and $y(t)$ are Fourier transform pairs, respectively. The step indexs explained in Section II-B1 is shown below the drawing.

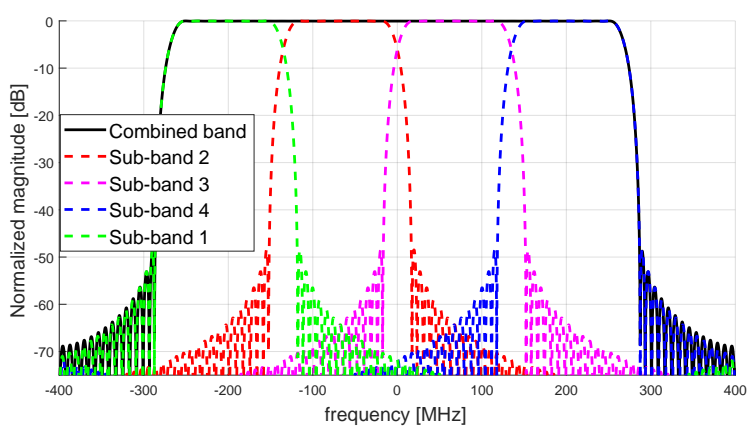

Figure 3. An illustration of the low-pass filter design. The parameter setting for the raised cosine filter is stop bandwidth $B_{\text {stop }}=170 \mathrm{MHz}$ and band spacing $B_{s}=135 \mathrm{MHz}$. The roll-off factor $\beta=\frac{B_{\text {stop }}}{B_{s}}-1=0.2593$, sampling interval is $T=\frac{1}{B_{s}}=7.4 \mathrm{~ns}$.

\section{EXPERIMENTAL VALIDATION}

A photo of the validation measurement setup is shown in Fig. 4. An analog signal generator is used to generate LO frequency. A vector signal generator and a signal analyzer are used to generate input RF signal and monitor the Doppler spectra. A sub-6 GHz CE with $160 \mathrm{MHz}$ bandwidth, which is equipped with 8 input ports and 8 output ports, is used. An RF down-converter and two 1-to- 8 RF splitter are integrated in one unit, while an RF up-converter and two 8-to-1 RF combiners are integrated in another unit, as shown in Fig. 4. A vector network analyzer (VNA) is utilized to record the emulated channel frequency response (CFR) emulated in the mmWave CE. It is noted that an RF cable is used between the analog signal generator and the VNA for the $10 \mathrm{MHz}$ reference connection. Off-the-shelf testing instruments are utilized to implement and validate the proposed mmWave CE scheme.

\section{A. Doppler Profile Validation}

Several channel models, including line-of-sight (LOS) models, non-LOS spatial cluster model and uniform Clark's model, are considered for demonstration purpose, as detailed in Table I. Delay profiles are not modeled in the Doppler validation measurement for simplicity. The channel fading coefficients are generated based on geometry-based stochastic channel modeling principle [15]. Doppler frequency specified in the

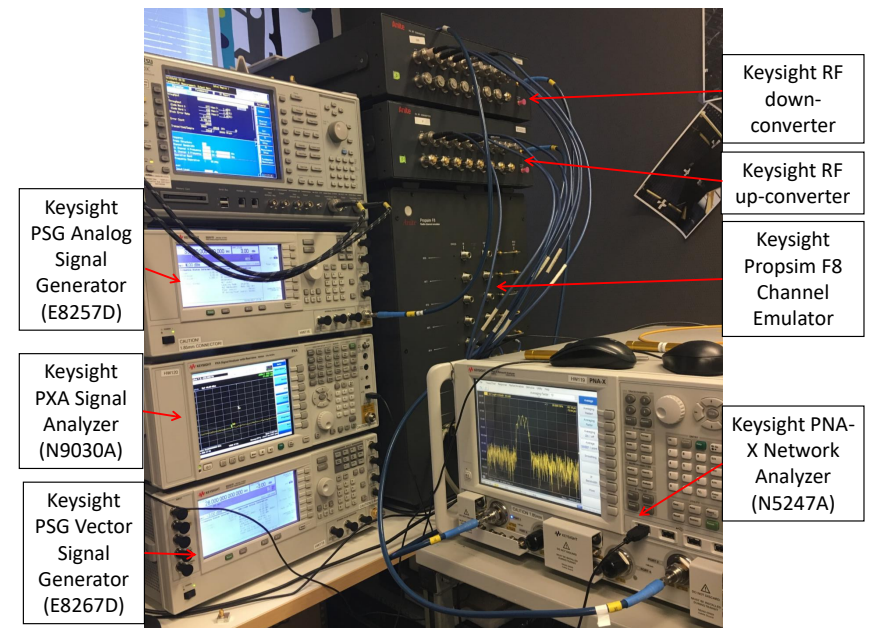

Figure 4. A photo of the measurement validation setup.

sub-6 GHz CE corresponds to the carrier frequency specified in the $\mathrm{CE}\left(f_{I F}=5.1 \mathrm{GHz}\right)$. The Doppler spectra will not be affected by the frequency up-and-down conversion. Therefore, to generate Doppler corresponding to the mmWave carrier frequency, we need to modify the $v_{I F}$ with a scaling factor in the sub-6GHz CE as:

$$
v_{I F}=\frac{f_{R F}}{f_{I F}} v_{R F}
$$

For example, to achieve $v_{R F}=100 \mathrm{~km} / \mathrm{h}$ and $v_{R F}=30$ $\mathrm{km} / \mathrm{h}$ at $28 \mathrm{GHz}$, we need to specify $v_{I F}=549 \mathrm{~km} / \mathrm{h}$ and $v_{I F}=164.7 \mathrm{~km} / \mathrm{h}$ at $5.1 \mathrm{GHz}$ in the sub- $6 \mathrm{GHz} \mathrm{CE}$, respectively, as shown in Table I.

Table I

CHANNEL PROFILE FOR DOPPLER PROFILE VALIDATION

\begin{tabular}{|c|c|}
\hline & Specifications in the sub-6 GHz CE \\
\hline A & Clarke's channel model, $v_{I F}=549 \mathrm{~km} / \mathrm{h} ;$ \\
\hline B & $\begin{array}{c}\text { A LOS path with direction of travel (DoT) } 0^{\circ}, \text { angle of arrival } \\
\text { (AoA) } 0^{\circ}, \text { speed of travel } v_{I F}=164.7 \mathrm{~km} / \mathrm{h} ;\end{array}$ \\
\hline C & A LOS path with DoT $0^{\circ}$, AoA $-45^{\circ}, v_{I F}=164.7 \mathrm{~km} / \mathrm{h} ;$ \\
\hline D & A LOS path with DoT $0^{\circ}$, AoA $-90^{\circ}, v_{I F}=164.7 \mathrm{~km} / \mathrm{h} ;$ \\
\hline E & $\begin{array}{c}\text { A non-LOS spatial cluster with Laplacian shape AoA }-135^{\circ} \\
\text { and azimuth spread (AS) } 5^{\circ}, \text { DoT } 0^{\circ} \text { and } v_{I F}=164.7 \mathrm{~km} / \mathrm{h} ;\end{array}$ \\
\hline
\end{tabular}




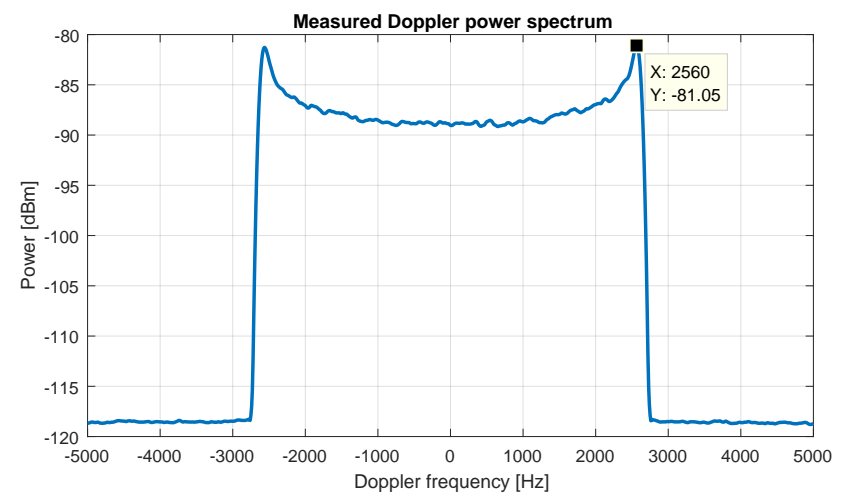

Figure 5. Measured Doppler profile for the Clarke's channel model.

The measured Doppler profile for channel model A is shown in Fig. 5. $f_{R F}=28 \mathrm{GHz}$ is shifted to $0 \mathrm{~Hz}$ for visualization purpose. The measured profile agrees well with the theoretical U shape for Clarke's Doppler profile, as expected. The maximum Doppler frequency $f_{R F}^{d}=\frac{v_{R F}}{c} f_{R F}=2593 \mathrm{~Hz}$, which agrees well with the measured value, as indicated in Fig. 5.

The simulated Doppler spectra can be obtained via performing Fourier transform of the time sequence of the CIR. For channel model B, C and D, a single Doppler frequency component at $f_{R F}^{d}=778 \mathrm{~Hz}, 550 \mathrm{~Hz}$ and $0 \mathrm{~Hz}$ are expected, respectively, as shown in Fig. 6 (top). The simulated Doppler spectra of channel B and D follow the ideal Dirac delta shape, while the spectrum of channel $\mathrm{C}$ only approximates the Dirac delta shape. This is due to the limited Doppler resolution in the Fourier transform. We can achieve more Dirac delta shape for channel $\mathrm{C}$ by increasing the number of time samples, which is set to 10000 in the simulations. The measured Doppler spectra for the considered channel models are shown in Fig 6 (below). As we can observe, the measured relative powers and Doppler profiles of the four channel models are well aligned with the corresponding simulated Doppler spectra. Note that the spreading and smoothing of measured Doppler spetra for each channel result from the resolution bandwidth setting in the spectrum analyzer. A narrower resolution bandwidth would offer sharper Doppler spectra measurement, which, however, would result in a longer sweeping time. It can be concluded from the Doppler spectra validation results that target Doppler profiles of the radio channels can be accurately emulated with the designed mmWave CE.

\section{B. Delay Profile Validation}

Four subchannels, each with $160 \mathrm{MHz}$, are utilized in the $\mathrm{CE}$ to achieve a target $500 \mathrm{MHz}$ system bandwidth. In the delay profile validation measurement, the Doppler profile is not modeled for simplicity.

1) Calibration stage: The calibration stage is essential in the band-combining scheme. To record the frequency response of each RF chain, we first need to bypass the channel for each sub-band. We firstly enable one CE sub-channel output port and disable the other three sub-channel output ports. Then we recorded the power level in the VNA. We can repeat the procedure to take down the power levels for each sub-band
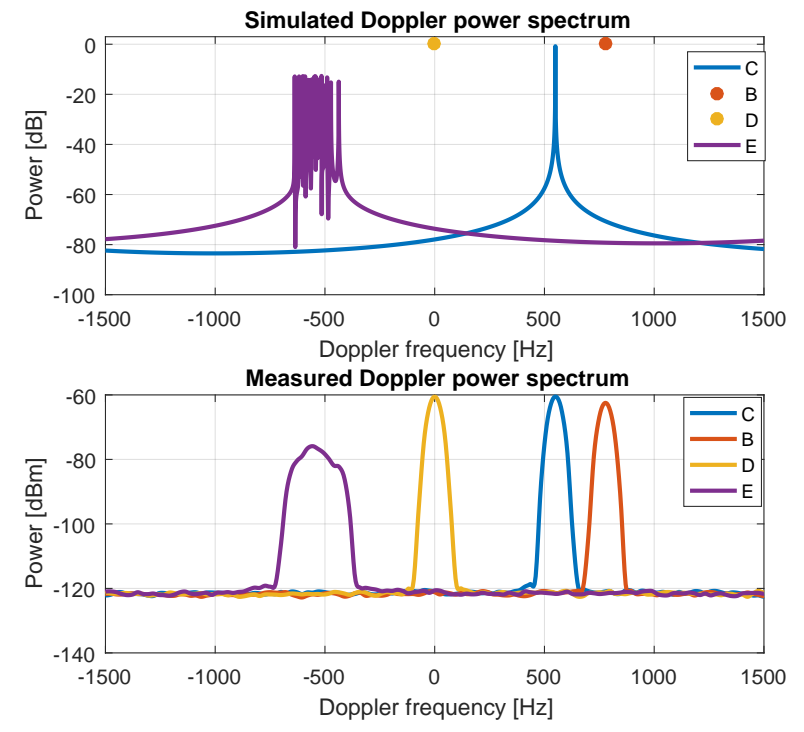

Figure 6. The simulated (top) and measured (below) Doppler profiles for channel model B, C, D, and E.

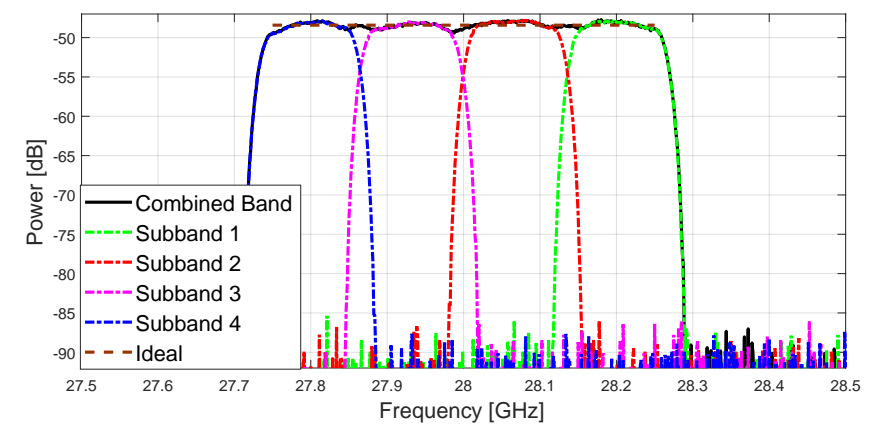

Figure 7. Measured frequency response of each subband and the full band after calibration with channel models bypassed.

channel. Then we can compensate the gain difference between sub-band RF links. After that, we enable all sub-band channel branches and tune the output port phase for each sub-band to ensure flat frequency response over the whole frequency band. The measured frequency responses of the RF chain associated with each sub-band and the full band after phase and gain calibration are shown in Fig. 7. The ripples (compared to the flat frequency response in the ideal case) over $500 \mathrm{MHz}$ is less than $\pm 1 \mathrm{~dB}$, which is mainly caused by the non-idealities in sub-6GHz CE. It is noted that $\pm 1.5 \mathrm{~dB}$ ripple is promised in the CE RF specifications [12].

The measured phase responses over frequency for each subband and the full band after calibration are shown in Fig. 8. In ideal case, a flat phase response over frequency is expected. However, the measured subband and full band phase responses are slanted linear curves within the frequency band, due to effective propagation length associated with each RF chain. The calibration works as expected, since same slope (indicating same propagation length) is achieved for different subbands and the full band after calibration.

2) Delay profile validation: After the calibration stage, the unequal frequency responses of different RF chains associated 


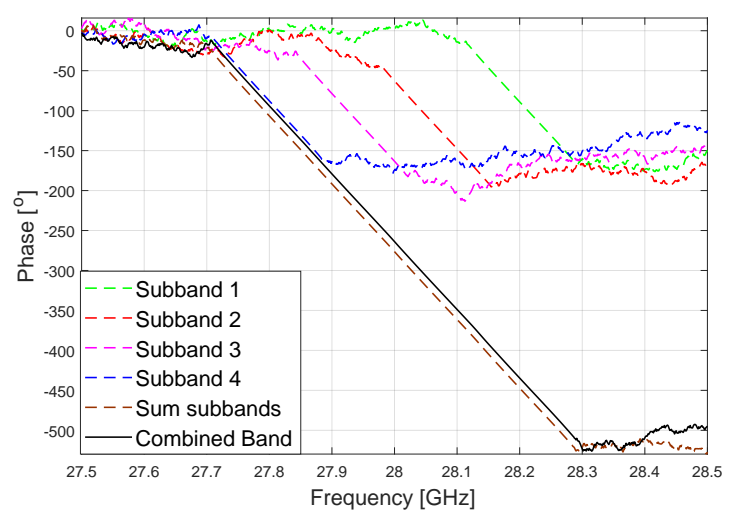

Figure 8. Measured phase of the frequency response for each subband and the full band after calibration with channel models bypassed.

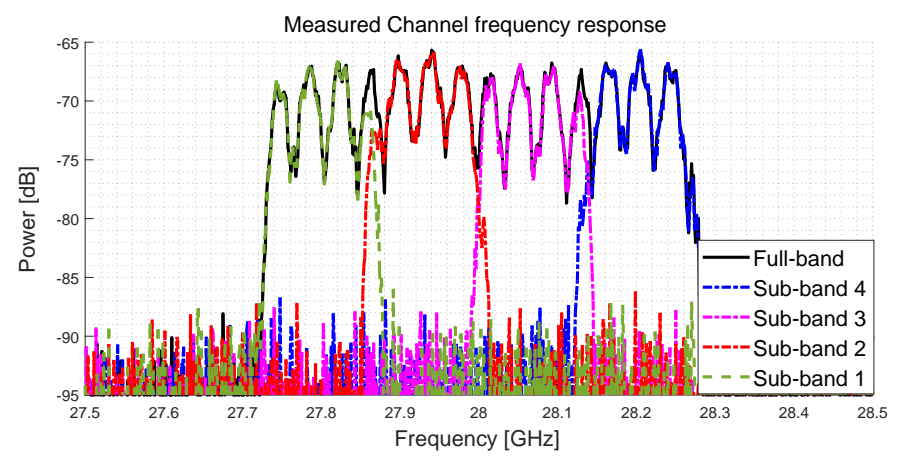

Figure 9. Measured frequency responses for each subband and the full band.

with sub-bands are compensated. To validate the delay profile emulation with a realistic channel model, a snapshot of METIS map-based model (i.e. a tap-delay-line with several delay taps) is utilized [16]. The measured CFRs for each sub-band and the full band are shown in Fig. 9. The sub-band CFR can be measured via enabling the respective RF chain and disabling all other three RF chains, while the full band CFR was recorded via enabling all four RF chains. The achieved dynamic range is around $30 \mathrm{~dB}$. The band-combining scheme works as expected, though small deviations can be observed in the transition regions between sub-bands. The target CFR over $500 \mathrm{MHz}$ band can be accurately achieved with four subband CFRs. The measured CIRs can be obtained via performing Fourier transform of the respective measured CFRs. A comparison between the measured CIR and target CIR in the METIS model is shown in Fig. 10. It is noted that both the the initial delay and the maximum power are normalized for the measured and simulated CIRs for comparison purpose. It can be seen that the measurement overlaps quite well with the target CIR. Compared with the measured sub-band CIR, the measured full-band CIR presents better delay resolution due to large achieved system bandwidth, as expected.

\section{Beamforming Validation}

As discussed, phase and gain tuning at the CE output ports are important for system calibration and OTA application purposes. There is a concern that phase control at mmWave

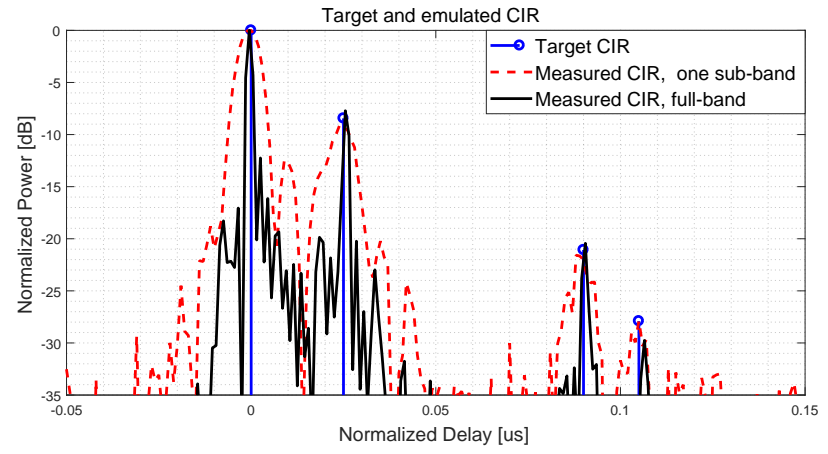

Figure 10. Target and measured channel impulse responses.

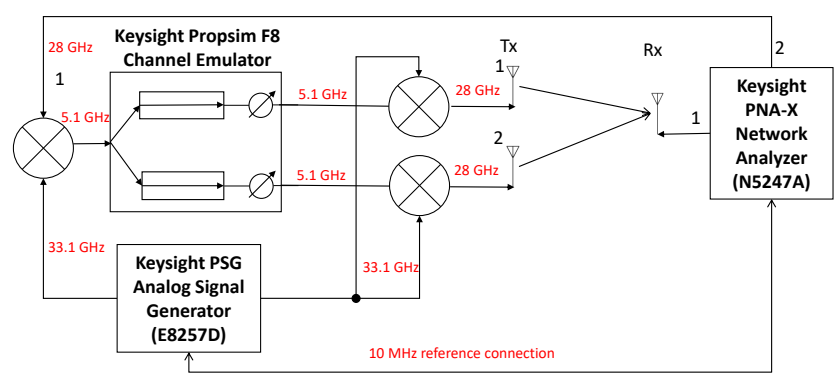

Figure 11. System setup for the beamforming validation measurement.

bands are challenging due to phase drifting and ambient temperature change. The phase and gain can be adjusted at the output port in the sub-6GHz CE, as illustrated in Fig. 1. In this section, the objective is to validate whether phase and gain control is accurate in the designed mmWave CE. The setup is illustrated in Fig. 11. Two channels (each with $160 \mathrm{MHz}$ bandwidth) are bypassed. A picture of the antenna placement is shown in Fig. 12. Unlike the previous measurements, the beamforming validation is performed over-the-air, as shown in Fig. 11.

In the measurement, a gain calibration was firstly performed, where the objective is to ensure same propagation loss for the two RF links associated with the two Tx antennas, respectively. In the gain calibration process, CE output port 1 was firstly disabled, with port 2 enabled. We recorded the transmission coefficient $s_{21}(f)$ in the VNA. After that, we repeated the same procedure with $\mathrm{CE}$ output port 2 enabled and output port 1 disabled. The gains at output ports were then modified to compensate out the gain difference between two RF links. After the gain calibration stage, both CE output ports are enabled. We can fix the phase term at one CE output as reference, and sweep the phase at the other CE output. For each swept phase within $\left[-200^{\circ}, 200^{\circ}\right]$, we recorded the the transmission coefficient $s_{21}(f)$ in the VNA. For a specific frequency, a peak and a null in the signal power are expected when sweeping the phase, which corresponds to constructive and destructive summation of signals (with equal gain) emitted from Tx 1 and Tx 2, respectively.

The measured frequency responses over swept phase term are shown in Fig. 13 (top), where a null in the power spectrum for a phase value can be observed. The simulated and 


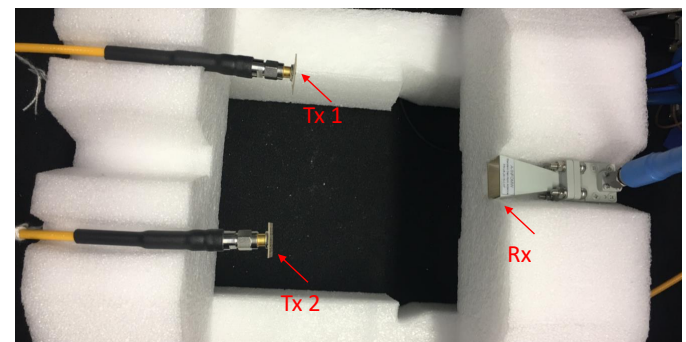

Figure 12. Picture of setup for beamforming validation. Patch antennas and horn antenna are used for the Tx and Rx antenna, respectively. Both Tx and $\mathrm{Rx}$ antennas are vertically polarized. Four patch antennas are utilized as an antenna element for each Tx antenna to improve the system dynamic range.
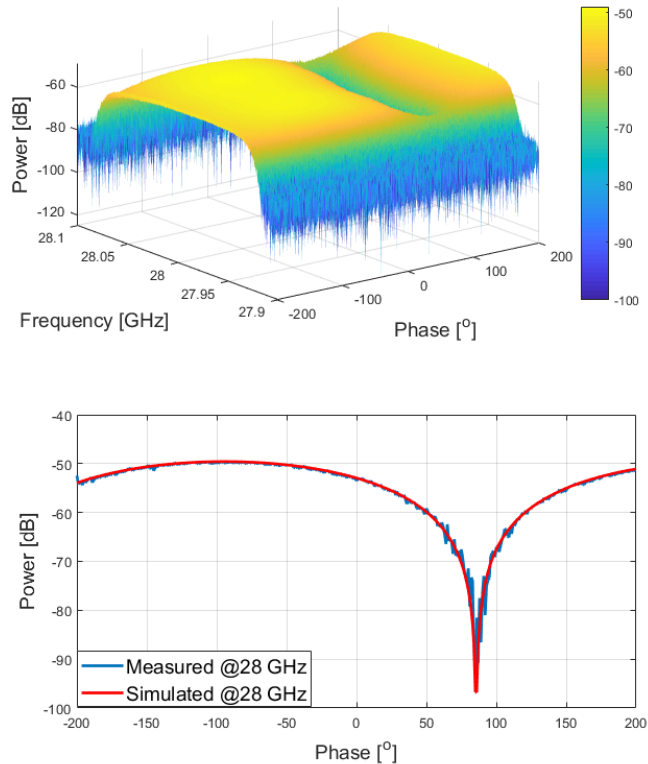

Figure 13. Measured frequency responses over $200 \mathrm{MHz}$ bandwidth with swept phase values (top) and measured and simulated power pattern over swept phase at $28 \mathrm{GHz}$ (below). The phase was swept over $\left[-200^{\circ}, 200^{\circ}\right]$ with $1^{\circ}$ step. A peak and a null exist at $-85^{\circ}$ and $95^{\circ}$, respectively.

measured power pattern over the swept phase at $28 \mathrm{GHz}$ are shown in Fig. 13 (below). It is noted that the signal amplitude level and phase difference determined in the measured data between two measured signals are utilized in the simulated curve for comparison. An excellent agreement between the measured and simulated curves is observed when the signal level is good, indicating an accurate phase and gain control capability of the output ports in the designed mmWave CE. Note that the deviation between measurement and simulation is large when the signal level is poor, due to the fact that power measurement is sensitive to noise when the signal level is low.

\section{CONCLUSION}

In this paper, a mmWave CE design, which supports flexible carrier frequency setting, flexible system bandwidth setting, and accurate phase and gain control at the output ports, is presented. The supported frequency range can cover both the sub-6 GHz and mmWave bands, with the help of frequency mixers. The system bandwidth can be flexibly set based on the proposed band-combining scheme, though we have to sacrifice $\mathrm{CE}$ resource to achieve more system bandwidth. Moreover, the mmWave CE design is based on current existing sub-6GHz CE. It is cost-effective, since only additional on-the-shelf frequency mixers, power splitters and combiners are needed to implement the mmWave CE. In the Doppler profile validation measurements, spatial channels, including specular LOS paths with different impinging angles, non-LOS spatial clusters and uniform Clark's model, are implemented in the mmWave CE. The measured Doppler profiles agree well with the target ones. In the delay profile validation measurements, the calibration results showed that the unequal frequency responses of RF chains associated with each sub-band can be compensated with phase and gain tuning. The measured results showed that the target channel frequency response can be accurately realized via four sub-band channel frequency responses, with the proposed band combining schemes. The over-the-air beamforming validation measurements also showed the accurate gain and phase control at the mmWave CE output ports.

\section{REFERENCES}

[1] "5G spectrum recommendation s," 5G Americas, Tech. Rep., April 2017.

[2] H. Eslami, S. V. Tran, and A. M. Eltawil, "Design and implementation of a scalable channel emulator for wideband mimo systems," IEEE Transactions on Vehicular Technology, vol. 58, no. 9, pp. 4698-4709, Nov 2009.

[3] W. Yu, Y. Qi, K. Liu, Y. Xu, and J. Fan, "Radiated Two-Stage Method for LTE MIMO User Equipment Performance Evaluation," IEEE Transactions on Electromagnetic Compatibility, vol. 56, no. 6, pp. 1691 1696, Dec 2014.

[4] CTIA, "Test plan for $r 2 \times 2$ downlink mimo and transmit diversity over-the-air performance," 2016.

[5] C. Schirmer, M. Lorenz, W. A. T. Kotterman, R. Perthold, M. H. Landmann, and G. D. Galdo, "MIMO over-the-air testing for electrically large objects in non-anechoic environments," in 2016 10th European Conference on Antennas and Propagation (EuCAP), April 2016, pp. $1-6$.

[6] W. Fan, P. Kyösti, L. Hentilä, and G. F. Pedersen, "Mimo terminal performance evaluation with a novel wireless cable method," IEEE Transactions on Antennas and Propagation, vol. 65, no. 9, pp. 4803 4814, Sept 2017.

[7] P. Kyosti and P. Heino, "Radio channel emulation," Jul. 30 2015, uS Patent App. 13/810,386. [Online]. Available: https://www.google.com/patents/US20150215053

[8] “ Real-time Massive MIMO Channel Emulation," NYU WIRELESS, Tech. Rep. [Online]. Available: http://wireless.engineering.nyu.edu/realtime-massive-mimo-channel-emulation/

[9] W. Fan, L. Hentilä, P. Kyösti, and G. F. Pedersen, "Test zone size characterization with measured mimo throughput for simulated mpac configurations in conductive setups," IEEE Transactions on Vehicular Technology, vol. PP, no. 99, pp. 1-1, 2017.

[10] “ ACE MX MIMO Channel Emulators ," Azimuth, Tech. Rep., June 2016.

[11] “ Wireless Channel Emulator: VR5 HD Spatial Channel Emulator ,' Spirent, Tech. Rep.

[12] "Propsim F8 Channel Emulator ," Keysight Technologies, Tech. Rep., August 2016.

[13] “ Vertex Channel Emulator ," Spirent, Tech. Rep. [Online]. Available: https://www.spirent.com/Products/Vertex-Channel-Emulator

[14] J. G. Proakis, M. Salehi, N. Zhou, and X. Li, "Communication systems engineering," 1994.

[15] "Spatial channel model for Multiple Input Multiple Output (MIMO) simulations," 3rd Generation Partnership Project, Tech. Rep. 3GPP TR 25.996 V13.0.0, December 2015.

[16] P. Kyösti, J. Lehtomäki, J. Medbo, and M. Latva-aho, "Map-based channel model for evaluation of $5 \mathrm{~g}$ wireless communication systems,' IEEE Transactions on Antennas and Propagation, vol. PP, no. 99, pp. $1-1,2017$. 